\title{
Nuclear correlation effects in neutrino-nucleus interactions
}

\author{
J. Marteau ${ }^{\mathrm{a}}$

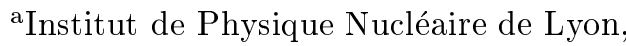 \\ IN2P3-CNRS and Université Claude Bernard, \\ 43 Bd du 11 Novembre 1918, \\ F-69622 Villeurbanne cedex, France
}

We present a model of neutrino-nucleus cross-sections computations taking into account nuclear effects such as short range correlations - through the ring approximation of the RPA, Delta resonance excitation with a medium modified decay width and $(n p$ - $n h)$ excitations $(n=2,3)$ relevant in the region between the quasi-elastic peak and the Delta peak. The inclusive reaction cross-section is split into exclusive channels classified according to the number of final state pions. This model was originally developped for the water Cerenkov experiments in which the pions in the final state often lead to Čerenkov ring(s) and are therefore crucial for the analysis.

\section{Introduction}

The uncertainties on the knowledge of the neutrino-nucleus interactions in the $\mathrm{GeV}$ region are becoming the strongest limitation in the analysis of the atmospheric neutrino experiments (such as Super-Kamiokande) and of the longbaseline experiments using low energy beams (K2K, MINOS (PH2le)).

The first point concerns the quasi-elastic peak. Its relevance is crucial since it is used in the water Cerenkov experiments as the main source of the "1 Čerenkov ring" events and in the long-baseline experiments for the neutrino events signature and beam knowledge in the close detector(s). This last feature is crucial if one wants to make an extrapolation of the event yields in the far detector. The questions are then : How are the quasi-elastic interactions modified in the medium (beyond the free nucleon or the Fermi gas approximation)? Are there some processes that could mimic the quasi-elastic events signature and what is their spectrum?

The second point concerns the pion production. In the resonance region around $\omega=M_{\Delta}-M_{N} \simeq 300 \mathrm{MeV}$ there is a strong modification of the spectrum with the appearance of strength between the quasi-elastic peak and the free Delta peak. This modification, referred to as the pionic branch was experimentaly seen with hadronic probes in the $\left({ }^{3} \mathrm{He}, t\right)$ reactions $[2,3]$. It is therefore worth evaluating how the leptonic neutrino probe can couple to this pionic branch and what is the impact of this coupling on the pion production. Furthermore the Delta width is also affected by the presence of the nuclear medium : distorsion of the emitted pion, existence of pionless decay channels (two-body $(2 p-2 h)$ and three-body $(3 p-3 h)$ absorption channels), Pauli blocking for the emitted nucleon. These features require a detailed parameterization and we will present the ingredients of our model.

The last point is the matching of the quasi-elastic and the Delta resonance region. Indeed it has been shown in the $\left(e, e^{\prime}\right)$ reactions that excitations of $(n p-n h)$ type can compensate the lack of strength in the "dip" region. The exact computation of such processes being quite heavy, we have adopted a more phenomenological approach in order to get a feeling of their importance.

The model developped offers the possibility to include all the mentionned nuclear effects up to the Delta resonance peak and also to include the residual interaction by the means of the ring approximation of the RPA. It is also possible to separate the total nuclear responses and the total cross- 
section into the various reaction channels. The model can therefore provide "exclusive" crosssections $\partial^{2} \sigma / \partial \Omega_{\text {lepton }} \partial p_{\text {lepton }}$ for the processes : $\nu_{\text {lepton }}\left(\bar{\nu}_{\text {lepton }}\right)+{ }^{A} X \longrightarrow$ lepton $^{-}\left(\right.$lepton $\left.{ }^{+}\right)+X^{\prime}$.

\section{Cross-sections computations}

The double differential cross-sections $\partial^{2} \sigma / \partial \Omega_{\text {lepton }} \partial k_{\text {lepton }}$ are computed as a linear combination of the nuclear responses which we will detail in the following section (details can be found in Ref.[1]). The lowest order in the expansion of the hadronic tensor in power of the target nucleon momentum leads to the following expression for the cross-section :

$$
\begin{aligned}
& \frac{\partial^{2} \sigma}{\partial \Omega_{\text {lep }} \partial p_{\text {lep }}}=\frac{G_{F}^{2} \cos ^{2} \theta_{C} \boldsymbol{p}_{l e p}^{2}}{2 \pi^{2}} \cos ^{2} \frac{\theta}{2} \times \\
& {\left[G_{E}^{2}\left(\frac{q^{2}}{\boldsymbol{q}^{2}}\right)^{2} R_{c}^{N N}+G_{A}^{2} \times\right.} \\
& \sum_{P, P^{\prime}=}\left(\frac{|\boldsymbol{q}|}{E_{q}+M}-\frac{\omega}{|\boldsymbol{q}|}\right)\left(\frac{|\boldsymbol{q}|}{E_{q}^{\prime}+M^{\prime}}-\frac{\omega}{|\boldsymbol{q}|}\right) R_{l l}^{P P^{\prime}}+ \\
& \left(G_{M}^{2} \frac{\boldsymbol{q}^{2}}{4 M_{N}^{2}}+G_{A}^{2}\right)\left(-\frac{q^{2}}{\boldsymbol{q}^{2}}+2 \tan ^{2} \frac{\theta}{2}\right) \sum_{P, P^{\prime}=} R_{t t}^{P P^{\prime}} \\
& \left. \pm 2 G_{A} G_{M} \frac{k+p_{l e p}}{M_{N}} \tan ^{2} \frac{\theta}{2} \sum_{P, P^{\prime}=} R_{t t}^{P P^{\prime}}\right]
\end{aligned}
$$

where $G_{F}$ is the weak coupling constant, $\theta_{c}$ the Cabbibo angle, $k$ and $p_{\text {lep }}$ the initial and final lepton momenta, $q=k-p_{\text {lep }}=(\omega, \boldsymbol{q})$ the 4-momentum transferred to the nucleus, $\theta$ the scattering angle, $E_{q}^{(\prime)}=\left(\boldsymbol{q}^{2}+M^{(\prime)}\right)^{1 / 2}$ with $M^{(\prime)}=M_{N}$ or $M_{\Delta}$ (nucleon or Delta mass). The plus (minus) sign in Eq.(1) stands for the neutrino (antineutrino) case. In this provisional approximation, we have neglected the lepton masses and we have kept the leading terms in the development of the hadronic current in $p / M_{N}$ where $p$ denotes the initial nucleon momentum. The electric, magnetic and axial form factors are taken in the standard dipole parameterization with the following normalizations: $G_{E}(0)=1.0, G_{M}(0)=4.71$ and $G_{A}(0)=1.25$.
We have introduced the nuclear response functions defined by :

$$
\begin{aligned}
& R_{\alpha \alpha}^{P P^{\prime}}=\sum_{n}\left\langle n\left|\sum_{j=1}^{A} O_{\alpha}^{P}(j) e^{i \boldsymbol{q} \cdot \boldsymbol{x}_{j}}\right| 0\right\rangle \times \\
&\left\langle n\left|\sum_{k=1}^{A} O_{\alpha}^{P^{\prime}}(k) e^{i \boldsymbol{q} \cdot \boldsymbol{x}_{k}}\right| 0\right\rangle^{*} \delta\left(\omega-E_{n}+E_{0}\right)
\end{aligned}
$$

where the particule-hole creation operators have the following forms :

$$
O_{\alpha}^{N}(j)=\tau_{j}^{ \pm}, \quad\left(\boldsymbol{\sigma}_{j} \cdot \widehat{q}\right) \tau_{j}^{ \pm}, \quad\left(\left(\boldsymbol{\sigma}_{j} \times \widehat{q}\right) \times \widehat{q}\right) \tau_{j}^{ \pm},
$$

for $\alpha \alpha=c c$ (charge), $\alpha \alpha=l l$ (spin-isospin longitudinal) and $\alpha \alpha=t t$ (spin-isospin transversal) respectively (nucleon case); and

$$
O_{\alpha}^{\Delta}(j)=\left(S_{j} \cdot \widehat{q}\right) T_{j}^{ \pm}, \quad\left(\left(S_{j} \times \widehat{q}\right) \times \widehat{q}\right) T_{j}^{ \pm},
$$

for $\alpha \alpha=l l$ (spin-isospin longitudinal) and $\alpha \alpha=$ $t t$ (spin-isospin transversal) respectively (Delta case). The longitudinal and transverse character of the spin-isospin operators refers to the direction of the spin operator with respect to the direction of the transferred momentum. The operators $S$ and $T$ are the usual 1/2 to $3 / 2$ transition operators in the spin and isospin space (for instance see [4]). In this work we neglect the small quadrupole transition connecting the nucleon to the Delta through the pure isospin operator, therefore the isospin response just involves nucleon-hole excitations. Note that we have assumed the existence of a scaling law between the nucleon and Delta magnetic and axial form factors [5]:

$$
G_{M}^{*} / G_{M}=G_{A}^{*} / G_{A}=f_{\pi N \Delta} / f_{\pi N N}=2.2,
$$

\section{Nuclear responses}

The evaluation of the nuclear responses is performed within the model developed by Delorme and Guichon for the interpretation of the $\left({ }^{3} \mathrm{He}, t\right)$ charge exchange experiments $[6,7]$.

\subsection{Formalism}

In this approach, a semi-classical approximation is performed and the nuclear responses are 
proportional to the imaginary part of the polarization propagator $\Pi$ which reduces to the well known Lindhard function [8] for a quasi-elastic nucleon-hole excitation in a Fermi gas. For instance :

$$
R(\omega, \boldsymbol{q})=-\frac{1}{\pi} \operatorname{Im}(\Pi(\omega, \boldsymbol{q}, \boldsymbol{q}))
$$

In the following we will denote $\Pi^{0}(\Pi)$ the polarization propagator without (with) the short range correlations.

The finite size effects are taken into account by the use of a local Fermi momentum $k_{F}(r)$ which is calculated by the means of an experimental nuclear density: $k_{F}(r)=\sqrt[3]{3 / 2 \pi^{2} \rho(r)}$. In the Delta-hole polarization propagators, the modified Delta width in the nuclear medium is split into the contributions of different decay channels [9]: the "quasi-elastic" channel $(\Delta \longrightarrow \pi N)$, modified by the Pauli blocking of the nucleon and the distorsion of the pion, the two-body ( $2 p$ $2 h)$ and three-body ( $3 p-3 h)$ absorption channels. These parameterizations lead to a good description of the pion-nucleus reactions (see for instance Fig.4). There are others (2p-2h) excitations which are not reducible to a modified Delta width and evaluated by extrapolating the calculations of two-body pion absorption at threshold [10]. This procedure is however more approximate and requires a detailed investigation in which mesonic exchange currents should be implemented.

\subsection{Partial reaction channels}

The Feynman graphs of the 7 reaction channels considered are shown in Fig.1 :

1. $N N$ quasi-elastic (Lindhard function),

2. $N N(2 p-2 h)$,

3. $N \Delta$ and $3^{\prime} . \Delta N(2 p-2 h)$,

4. $\Delta \Delta(\pi N)$,

5. $\Delta \Delta(2 p-2 h)$,

6. $\Delta \Delta(3 p-3 h)$.

The total polarization propagator $\Pi^{0}$ is the sum of all the partial reaction channels : $\Pi^{0}=\sum_{k} \Pi_{(k)}^{0}$. This division allows to

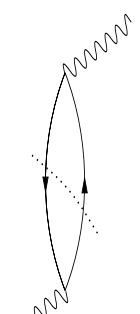

(1)

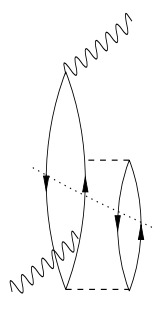

(2)

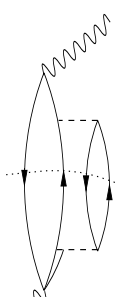

(3)

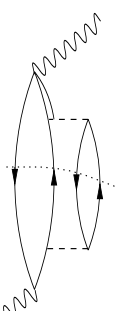

(3')
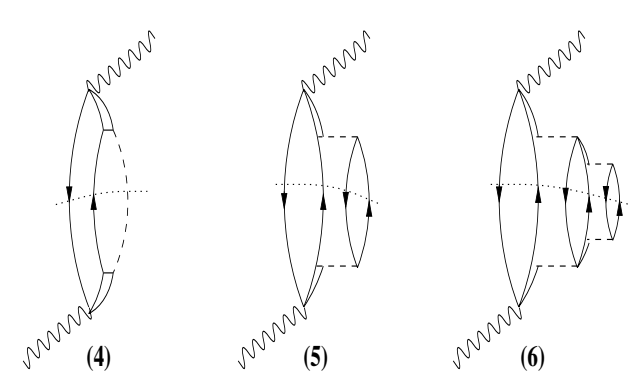

Figure 1. Feynman graphs of the partial polarization propagators: $N N$ quasi-elastic (1), $N N(2 \mathrm{p}-2 \mathrm{~h})(2)$, $N \Delta(2 \mathrm{p}-2 \mathrm{~h})(3), \Delta N(2 \mathrm{p}-2 \mathrm{~h})\left(3^{\prime}\right), \Delta \Delta(\pi N)(4), \Delta \Delta$ (2p-2h) (5), $\Delta \Delta$ (3p-3h) (6). The wiggled lines represent the external probe, the double lines the Delta resonance, the dashed line the pion and the full line the nucleon. The dotted line indicates which lines are put on-shell in order to get the response (imaginary part of the polarization propagator).

make an "exclusive" analysis of the results by computing the double differential crosssections (Eq.1) with the partial responses : $R_{(k)}^{0}(\omega, \boldsymbol{q})=-\frac{1}{\pi} \operatorname{Im}\left(\Pi_{(k)}^{0}(\omega, \boldsymbol{q}, \boldsymbol{q})\right)$. This exclusive analysis is still valid when the correlations between particule-hole states are taken into account.

\subsection{Residual interaction}

The short range correlations indicate how a particule-hole state initiated by an external probe (for instance a neutrino) can propagate into the medium. This propagation is described by the means of an interaction potential. In this model we exactly solve the RPA equations in the ring-approximation. The initial polarization 
propagator $\Pi^{0}$ is used as an input to solve : $\Pi=\Pi^{0}+\Pi^{0} V \Pi$. The interaction potential $V$ is parameterized by a pure contact force (with a parameter of Landau-Migdal $f^{\prime}=0.6$ ) in the isospin channel and by a $(\pi+\rho)$-exchange plus a contact force (Landau-Migdal parameter $g^{\prime} \in[0.5,0.7]$ ) in the spin-isospin channel :

$$
\begin{aligned}
& V_{N N}=\left(f^{\prime}+V_{\pi}+V_{\rho}+V_{g^{\prime}}\right) \boldsymbol{\tau}_{1} \cdot \boldsymbol{\tau}_{2} \\
& V_{N \Delta}=\left(V_{\pi}+V_{\rho}+V_{g^{\prime}}\right) \boldsymbol{\tau}_{1} \cdot \boldsymbol{T}_{2}^{\dagger} \\
& V_{\Delta N}=\left(V_{\pi}+V_{\rho}+V_{g^{\prime}}\right) \boldsymbol{T}_{1} . \boldsymbol{\tau}_{2} \\
& V_{\Delta \Delta}=\left(V_{\pi}+V_{\rho}+V_{g^{\prime}}\right) \boldsymbol{T}_{1} . \boldsymbol{T}_{2}^{\dagger}
\end{aligned}
$$

where in the $N N$ case, for example (the $N \Delta, \Delta N$ and $\Delta \Delta$ cases are obtained with the appropriate replacements $\sigma \longrightarrow S$ ):

$$
\begin{aligned}
V_{\pi} & =F_{\pi}^{2}\left(\frac{\boldsymbol{q}^{2}}{\omega^{2}-\boldsymbol{q}^{2}-m_{\pi}^{2}}\right)\left(\boldsymbol{\sigma}_{1} \cdot \widehat{q}\right)\left(\boldsymbol{\sigma}_{2} \cdot \widehat{q}\right) \\
V_{\rho} & =F_{\rho}^{2}\left(\frac{\boldsymbol{q}^{2}}{\omega^{2}-\boldsymbol{q}^{2}-m_{\rho}^{2}}\right)\left(\boldsymbol{\sigma}_{1} \times \widehat{q}\right) \cdot\left(\boldsymbol{\sigma}_{2} \times \widehat{q}\right) \\
V_{g^{\prime}} & =F_{\pi}^{2} g^{\prime} \boldsymbol{\sigma}_{1} \cdot \boldsymbol{\sigma}_{2}
\end{aligned}
$$

In the preceding equations, $F_{\pi}(q)$ and $F_{\rho}(q)$ are the standard pion-nucleon and rho-nucleon form factors.

The exclusive RPA response functions are obtained by taking the imaginary part of the RPA polarization propagators. This leads to 8 partial response functions :

$$
\begin{aligned}
R_{(k)}(\omega, q)= & -\frac{1}{\pi}|1+\Pi V|^{2} \operatorname{Im}\left(\Pi_{(k)}^{0}(\omega, q, q)\right) \\
& \text { for } \mathrm{k}=1, \ldots, 7 \\
R_{c o h}(\omega, q)= & -\frac{1}{\pi}|\Pi|^{2} \operatorname{Im} V
\end{aligned}
$$

The last response function corresponds to the "coherent" emission of a pion on its mass shell (in the domain of energy considered here the sole contribution to this channel comes from the pion exchange).

\subsection{Results}

The spin-isospin (longitudinal and transverse) responses functions are displayed as a function of transfer energy for a fixed momentum value $(200$ $\mathrm{MeV} / \mathrm{c}$ ) in Fig.2. Without RPA both responses functions are identical (dotted curve). They exhibit two characteristic peaks (quasi-elastic peak

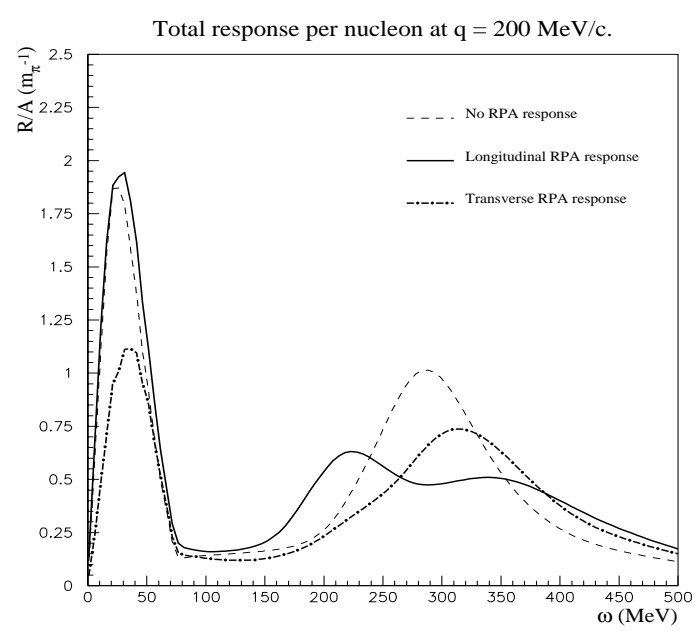

Figure 2. Total nuclear responses vs transfer energy at fixed momentum $(q=200 \mathrm{MeV} / \mathrm{c})$ without RPA (dotted line) and with RPA : longitudinal (bold line) and transverse (dot-dashed line).

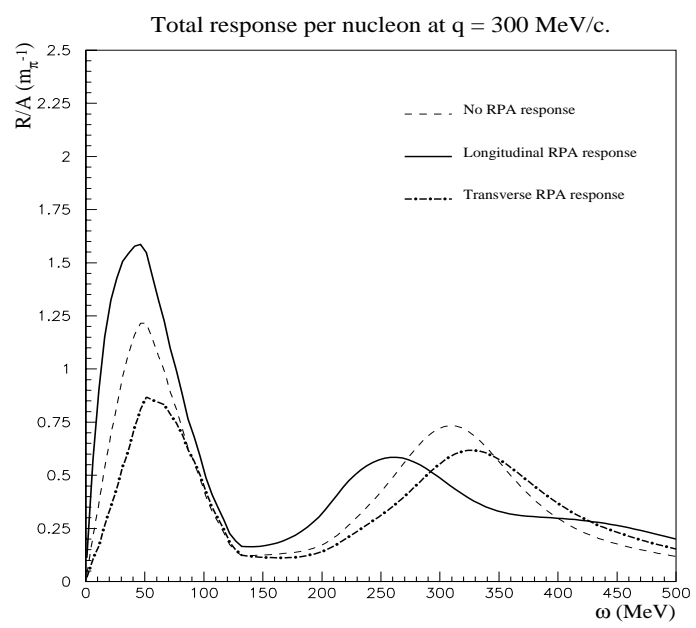

Figure 3. Same plot as Fig.2 at $q=300 \mathrm{MeV} / \mathrm{c}$. 
at low energy and Delta peak at higher energy). The strength in the "dip" region is provided by the $n p-n h$ excitations. The residual interaction distinguishes between the longitudinal channel (which is coupled to the pion) and the transverse channel (coupled to the rho). Indeed in the longitudinal channel the interaction potential becomes attractive (the pion exchange compensates the repulsive part of the potential given by $g^{\prime}$ ) at low momenta (around $200 \mathrm{MeV} / \mathrm{c}$ ) due to the smallness of the pion mass. In the transverse channel the potential remains repulsive over a large domain of momentum.

A repulsive interaction potential will harden the spectrum and decrease the response function. This clear in Fig.2. In the longitudinal channel the spectrum is strongly affected in shape by the RPA. The first modification concerns the quasielastic which is enhanced w.r.t. the non RPA situation (Fig.3). The second affects the Delta peak which is splitted into two branchs : the higher energy branch is the modified Delta branch and the lower energy one is the pionic branch (Fig.2). At $q=300 \mathrm{MeV} / \mathrm{c}$ the Delta branch almost disappears and the pionic branch concentrates all the strength (Fig.3).

\subsection{Comparison with experiment}

The model presented in this paper has been tested over a wide range of experimental data. As mentionned before it was developped for the $\left({ }^{3} \mathrm{He}, t\right)$ charge exchange experiments where it gives satisfactory results. A direct comparison with experimental data can be done in the $\pi$ nucleus reactions. An example is given in Fig.4 which displays the total cross-section (directly proportionnal to the longitudinal response calculated on the pion mass shell) for different kind of nuclei. The agreement shown is globally good except for the higher energy region where there is a constant (with $A$ ) lack of strength. This can be compensated by integarting in the calculation more terms in the $p / M_{N}$ expansion.

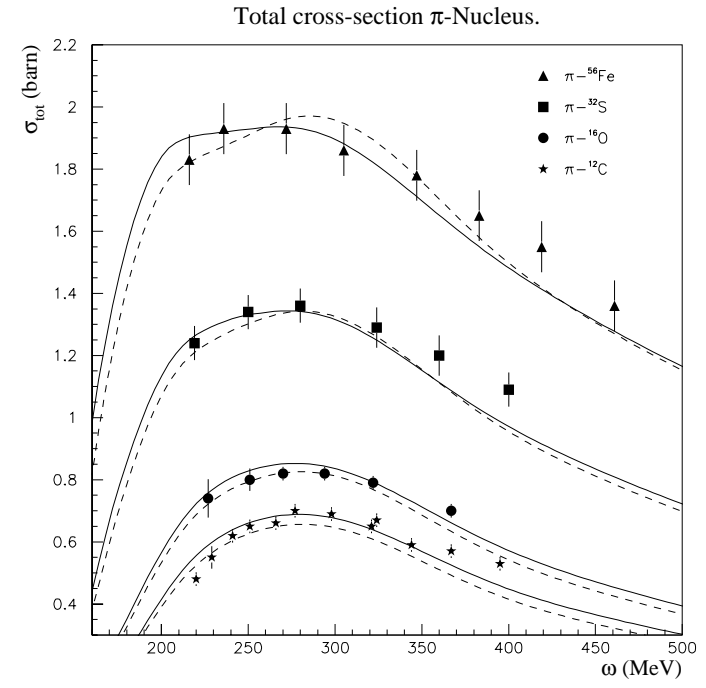

Figure 4. Total $\pi$-nucleus cross-sections computed with the semi-classical approach presented in the text.

\section{Impact on the neutrino reactions}

\subsection{Inclusive cross-sections}

The general features of the short range correlation are :

- Suppression of the longitudinal QuasiElastic response at leading order (this is obvious in Eq.1 if one considers that the quasi-elastic response peaks around the line $\omega=q^{2} / 2 M_{N}$ and if one keeps the leading order terms in $\left.|\boldsymbol{q}| / M_{N}\right)$. This exact cancellation at the quasi-elastic peak is of kinematical origin. The suppression of the longitudinal channel is less important in the anti-neutrino case where the weight of the transverse channel is smaller (change of sign in the interference term).

- Dominance of the transverse spin-isospin channel. This implies that the pionic branch will have little effect and that the hardening of the transverse response provides the main modifications. 


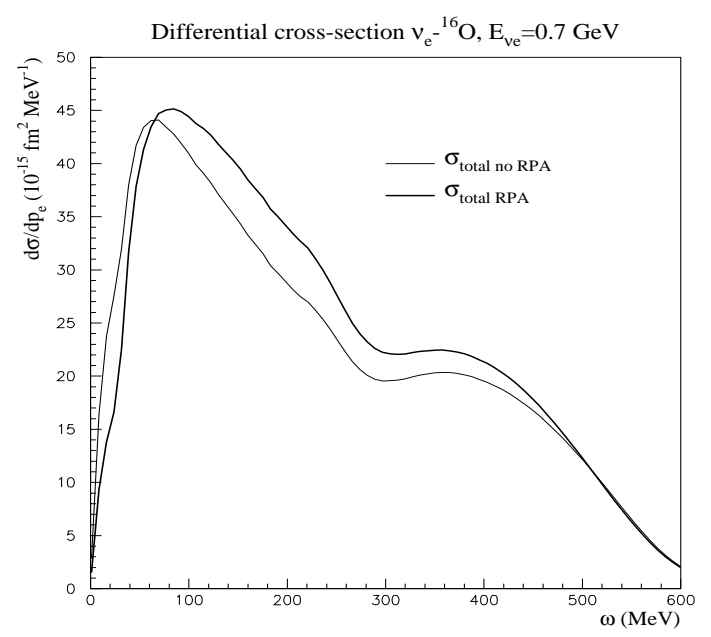

Figure 5. Differential cross-section $\partial \sigma / \partial E_{e}$ for incident $\nu_{e}$ 's with an energy of $0.7 \mathrm{GeV}$.

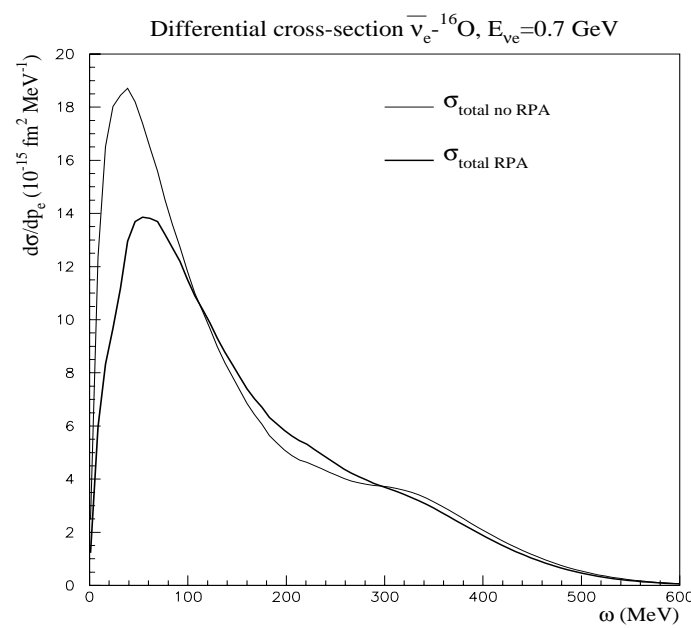

Figure 6. The same plot as Fig.5 for anti- $\nu_{e}$ 's.

These effects of the RPA on the differential crosssections $\partial \sigma / \partial E_{e}$ for incident $\nu_{e}$ 's (and anti- $\nu_{e}$ 's) with an energy of $0.7 \mathrm{GeV}$ are shown in Fig.5 (Fig.6). The bold (thin) lines are for the RPA (free) cases. The hardening of the cross-section is clear for neutrinos $(\sim 40 \mathrm{MeV}$ shift of the quasielastic peak towards higher energy). For antineutrinos the decrease of the quasi-elastic peak and the suppression of the Delta peak are the two expected characteristics.

\subsection{Exclusive cross-sections}

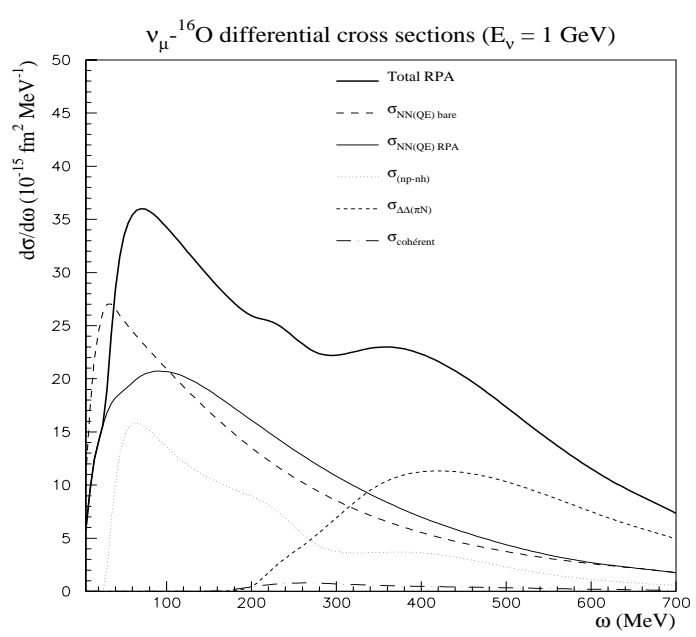

Figure 7. $\nu_{\mu}-{ }^{16} \mathrm{O}$ charged-current reactions: differential cross-section vs energy transfer.

Fig.7 shows the exclusive differential crosssections $\partial \sigma / \partial E_{\mu}$ for $\nu_{\mu}$ 's of $1 \mathrm{GeV}$ vs the transfer energy. The inclusive cross sections (thick curves) get its main contribution from the quasielastic channel (thin full curve) which peaks at low energy transfer. For a sake of comparison we have shown the contribution of the quasi-elastic channel without RPA (thin long dashed line). The hardening due to the RPA is very clear in this channel. Another very interesting feature is the importance of the $(n p-n h)$ channels (dotted curve) which give a large contribution to the inclusive cross sections in the "dip" region. 


\subsection{Neutrino events rates}

Having computed the cross section we are in position to evaluate the neutrino-oxygen events yields at fixed charged lepton momentum $Y\left(k^{\prime}\right)=\int d E_{\nu} \Phi_{\nu} \partial \sigma / \partial k^{\prime}$ where $\Phi_{\nu}$ the incoming neutrinos flux taken from ref. [11]. Fig.8 gives the results for the "pionless" events (quasi-elastic + $n p-n h$ : full curves, quasi-elastic alone : dashed curves).

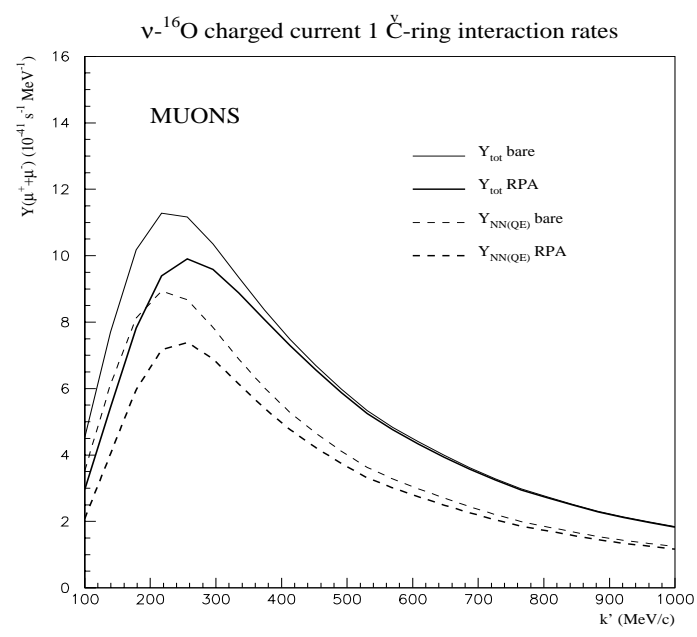

Figure 8. $\nu_{\mu}-{ }^{16} \mathrm{O}$ quasi-elastic $+n p$ - $n h$ (full curves) and quasi-elastic alone (dashed curves) events yields vs muon momentum. The thick curves are for the RPA calculation whereas the thin curves are for the non RPA calculation.

The RPA tends to reduce the events yields. Indeed it shifts the cross section towards high energies which are disfavored by the neutrino flux. In one now includes the $n p$ - $n h$ reaction channels in the same events category (with no pion in the final state) we see an enhacement of the total yield with respect to the free quasi-elastic around 20 $\%$. This result points out the importance of a good evaluation of such neutrino induced $n p-n h$ excitations.

\subsection{Exclusive $\Delta$ reaction channels}

We conclude this work by mentioning the problem of pion emission in neutrino-oxygen interactions. On one side we have shown that the cross sections of the $\Delta \Delta(2 p-2 h)$ and $(3 p-3 h)$ partial channels, which do not not lead to pion emission (non pionic channels), extend over a broad region in transfer energy, while the pionic channel $\Delta \Delta$ $(\pi N)$ is peaked at high transfer energy (see Fig. (7)). On the other side the neutrino flux lowers the weight of the high energies and favors the low energy components of the spectrum. Then the pionic $\Delta \Delta$ channel will be more suppressed by the incident neutrino flux than the non pionic one. This result is shown on fig. (9) where the total $\Delta \Delta$ events yield (full thick curve) is splitted into its three contributions: $(\pi N)$ (full thin curve), $2 p$-2h (dashed curve), $3 p$-3h (dotted curve) in the case of $\mu$-type events. The main result is that the fraction of the non pionic channels over the pionic one is around $50 \%$. This confirms the importance of a good evaluation of the $n p-n h$ excitations in the medium.

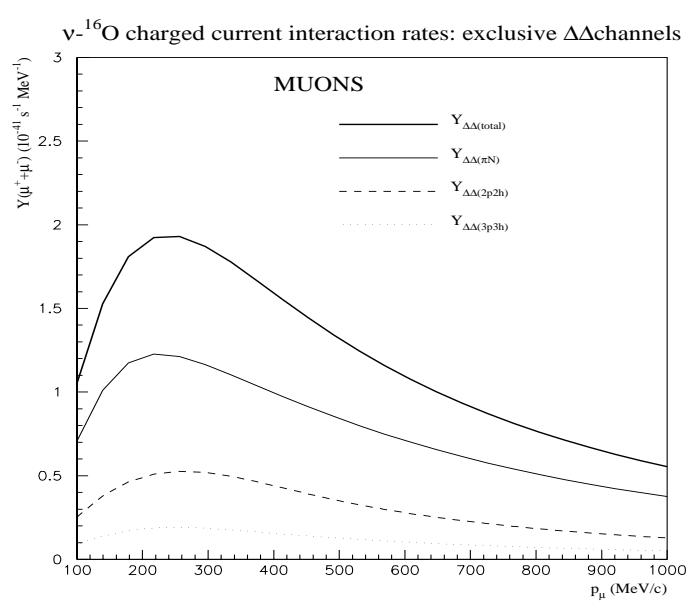

Figure 9. Contributions to the total $\Delta \Delta$ events yield (full thick curve) of the partial channels: $(\pi N)$ (full thin curve), (2p-2h) (dashed line), (3p-3h) (dotted line). 


\section{Conclusion}

In this work we studied the role of nuclear physics in the neutrino-nucleus reactions with a particular emphasis for the neutrino-oxygen reactions relevant for the atmospheric neutrinos Cerenkov detectors. The model presented offers the possibility to include the effects of residual interaction, $n p$ - $n h$ excitations (reducible and non reducible to a Delta width), modified Delta width in the medium, finite size of the nucleus. The semi-classical approach adopted leads to a flexible and unified description of these processes from the quasi-elastic peak to the Delta peak.

It leads to a possible analysis of the exclusive reaction channels, classified for example w.r.t. the presence or not of pions in the final state. We conclude that the neutrino-nucleus cross-section is strongly dominated by the transverse channel in which the effects of the residual interaction is not as important than in the longitudinal channel. This leads mainly to a hardening of the crosssections and therefore a decrease of the atmospheric neutrinos event rates due to the sharp decrease with neutrino energy of the neutrino flux. The modified Delta width in the medium leads to a consequent modification of the number of pions emitted when compared to the free case. This work points out the need of a detailled evaluation of the $n p-n h$ excitations in particular.

\section{REFERENCES}

1. J. Marteau, Eur. Jour. J. A5, 183-190(1999).

2. D. Contardo et al., Phys. Lett. B168, 331(1986).

3. V.G. Ableev et al., J.E.T.P. Lett. 40, 763(1984).

4. T. Ericson, W. Weise, Pions and nuclei, Oxford Science Publications, 1988.

5. G.F. Low, et al, Phys. Rev. 106, (1957) 1345.

6. J. Delorme, P.A.M. Guichon, in Proceedings of $10^{e}$ biennale de physique nuclaire, Aussois, march 6-10, 1989, rapport LYCEN 8906, p. C.4.1, also in the Proceedings of the 5th french-japanese symposium on nuclear physics, Dogashima, Izu, september 2630, 1989, edited by K. Shimizu and O.
Hashimoto, p.66.

7. J. Delorme, P.A.M. Guichon, Phys. Lett. B 263, 157(1991).

8. A.L. Fetter, J.D. Walecka, Quantum theory of many-particle systems, Mc Graw-Hill Book Company, 1971.

9. E.Oset, L.L.Salcedo, D.Strottman, Phys. Lett. 165B, 13(1985).

10. K.Shimizu, A.Faessler, Nucl. Ph. A333, 495(1980).

11. G.Barr, T.K.Gaisser, T.Stanev, Phys. Rev. D39, 3532(1989). 